\title{
X-RAY POWDER DIFFRACTION IN THE ACTIVITIES OF THE LABCACC
}

\author{
Antonio, S. G. ${ }^{1, *}$; Salvi, S. T. B. ${ }^{2}$; Tita, D. L. ${ }^{1}$; Paiva-Santos, C.O. ${ }^{1}$ \\ ${ }^{1}$ Department of Physical Chemistry, UNESP, Institute of Chemistry, Araraquara, São Paulo, Brasil \\ *Selma_ga@yahoo.com.br
}

During the last 20 years $\mathrm{x}$-ray powder diffraction are being applied in our Laboratory to study pharmaceuticals ingredients and their products and in ceramics. In addition, we have dedicated a great effort in the teaching area, giving Rietveld and crystallographic courses in Universities, scientific events and industries (nearly three dozen in the last five years). Recently, the PONKCS method described by Scarlet and Madsen has been applied to the quantitative phase analysis (QPA), also in raw materials of pharmaceuticals and their products. Initially we realized that the PONKCS method provides better results than those obtained with the Rietveld method, especially when the atomic displacements are not provided with the CIF files. After some testing, we found that the fraction of a particular drug, as determined by RM, provides different results depending on the region of 2 measured, while with PONKCS it does not happens. With RM, to refine the atomic displacements in a pharmaceuticals can lead to serious problems in the QPA due to anisotropy, preferred orientation of the crystallites and high overlap of peaks caused by the low symmetry of the phases involved. With PONKCS it is not necessary to refine atomic displacement and this problem does not occur. This year, to celebrate 100 years of Crystallography, we are developing a course online, Characterization of organic and inorganic materials by diffraction of X rays by polycrystalline. We will present examples of PONKCS in pharmaceuticals and describe the online course. 